\title{
TUTORIAL CONVIDADO \\ PESQUISA OPERACIONAL EM ÉPOCA DE CONFLITOS ARMADOS, PANDEMIAS E CATÁSTROFES NATURAIS ${ }^{1}$
}

\author{
Nelson Maculan ${ }^{\mathrm{a} *}$, Laura Bahiense ${ }^{\mathrm{b}}$

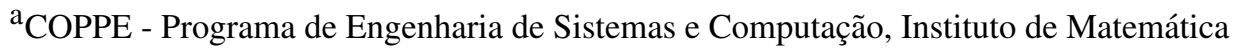 \\ Universidade Federal do Rio de Janeiro - UFRJ, Rio de Janeiro-RJ, Brasil \\ ${ }^{\mathrm{b}}$ COPPE - Programa de Engenharia de Sistemas e Computação, Programa de Engenharia de Transportes \\ Universidade Federal do Rio de Janeiro - UFRJ, Rio de Janeiro-RJ, Brasil
}

Recebido 04/08/2020, aceito 04/08/2020

\section{RESUMO}

Neste artigo, discutimos problemas relacionados a perdas humanas em conflitos armados, pandemias e catástrofes naturais, e o papel de Pesquisa Operacional visando a minimizar essas perdas.

Palavras-chave: Pesquisa operacional, Conflitos armados, Pandemias, Catástrofes naturais.

\begin{abstract}
In this article, we discuss problems related to human losses in armed conflicts, pandemics and natural catastrophes, and the role of Operational Research to minimize these losses.
\end{abstract}

Keywords: Operations research, Armed conflicts, Pandemics, Natural catastrophes.

*Autor para correspondência. E-mail: maculan@ cos.ufrj.br

DOI: 10.4322/PODes.2021.001

${ }^{1}$ Todos os autores assumem a responsabilidade pelo conteúdo do artigo. 


\section{Introdução}

Estamos passando pela pandemia da COVID-19, causada pelo coronavírus SARS-CoV-2. Além disso, vários conflitos armados regionais criam condições insuportáveis de vida para a população civil, muitas vezes obrigada a imigrar e a viver em acampamentos com milhares de pessoas. Sem falar das catástrofes naturais, que vêm crescendo nos últimos anos em todo o mundo.

Operações logísticas eficientes são essenciais para o sucesso do socorro humanitário, seja em pandemias, conflitos armados ou situações de catástrofe natural. A cadeia de suprimentos humanitários precisa fornecer respostas rápidas aos eventos imprevisíveis, levando em conta as diversas limitações operacionais (orçamentárias, de pessoal, de acesso etc.) e gerenciais (governos, agências de ajuda, doadores, voluntários, ONGs etc.).

Nesse contexto, os problemas de localização costumam ser muito utilizados. Por exemplo, a localização dos postos avançados de logística é questão chave para o bom funcionamento da logística humanitária, já que possuem a função de orientar a população, prover remédios e outros insumos, e centralizar os profissionais e voluntários capacitados.

Outra questão importante envolve a decisão sobre a localização dos armazéns de suprimentos, já que o tempo de resposta do atendimento às populações atingidas deve ser o menor possível, contudo a infraestrutura dos locais impactados costuma ser precária, e deve-se contornar, ainda, a limitação de recursos.

Os trabalhos Nagurney (2020), Díaz et al. (2019), Marinho et al. (2018), Maculan-Guido (2017), Fereiduni et al. (2016), Camacho-Vallejo et al. (2015), Brito Jr. (2015) tratam pontos em comum com os assuntos que serão aqui abordados.

\section{Conflitos Armados}

O trabalho Monemi et al. (2020) servirá de base para esta seção. Nele, abordamos o problema da distribuição de ajuda humanitária nos campos de refugiados em locais de guerra. Mostramos que o problema pode ser modelado como uma variante do problema de localização multiperíodo com um padrão de demanda específico resultante do comportamento do usuário.

O problema foi motivado por um estudo de caso da experiência libanesa em acomodações de refugiados da guerra na Síria. Levando em consideração a complexidade e as restrições da vida real, propusemos uma formulação através de um modelo matemático de otimização (Programação Linear Inteira Mista) para o problema. Para a sua solução, usamos a decomposição de Benders e conseguimos encontrar desigualdades válidas, assim como os algoritmos de separação. Nossos extensos experimentos computacionais com o estudo de caso com dados reais e instâncias geradas aleatoriamente comprovaram o bom desempenho do método de solução proposto.

As autoridades libanesas dividiram o Líbano em 26 distritos em centróides de ciclos, cujos diâmetros são proporcionais à população dos sírios exilados residindo nessas regiões. Mais de $70 \%$ dos sírios exilados vivem abaixo do nível de pobreza.

O objetivo principal do artigo consistiu em propor uma rede visando à distribuição de auxílios humanitários no caso dos refugiados sírios no Líbano, de forma a melhorar a qualidade dos serviços prestados pelo ACNUR (Alto Comissariado das Nações Unidas para os Refugiados), garantindo a frequência do serviço e satisfazendo as demandas em tempo hábil.

As demandas dos campos eram semanais e deveriam ser distribuídas em até 5 dias. Alguns campos podiam receber tudo no mesmo dia, outros em dois dias ou ainda em três dias. Para cada campo havia uma frequência de entrega. Por exemplo, frequência de um só dia - $100 \%$ da demanda em uma única entrega; frequência de dois dias - 30\% na segunda-feira e $70 \%$ na quarta feira; frequência de três dias - 35\% na terça-feira, $25 \%$ na quarta-feira e $40 \%$ na sexta-feira.

Todo o transporte era feito por caminhões com capacidades próximas. Era conhecido o número máximo de caminhões que poderiam ser utilizados em cada dia. Cada caminhão saía do depósito principal durante à noite para um centro de distribuição, situado em um dos campos de refugiados. No dia seguinte, parte dos caminhões era descarregada para satisfazer a demanda dos 
campos de refugiados onde foram escolhidos os centros de distribuição, e os demais caminhões deixavam os centros de distribuição para atender as demandas dos demais campos de refugiados. Todos os caminhões já vazios retornavam até o final da tarde ao depósito principal para serem carregados visando a suprir a demanda do dia seguinte. De acordo com a demanda diária e a frequência das entregas, o número de centros de distribuição e suas localizações podem variar.

O modelo matemático proposto objetivou prover uma solução que minimizasse o custo total de transporte, os custos fixos de instalação dos centros de distribuição e o número de caminhões, satisfazendo todas as demandas que variam diariamente, e determinando os melhores percursos dos caminhões. Trata-se de um problema de otimização combinatória.

Dada a complexidade computacional do problema (NP-difícil), nem sempre conseguimos encontrar uma solução ótima, mas algumas soluções viáveis e uma cota inferior para a função objetivo são fornecidas durante a execução do algoritmo. A melhor solução encontrada durante a enumeração implícita de soluções viáveis do algoritmo de otimização utilizado (branch-and-cut), com ou sem a ajuda de metaheurísticas, pode ser uma boa solução para o problema real.

Na realidade, estamos em presença de um problema de percurso (roteamento) dinâmico, em que as demandas variam a cada dia. E a novidade do modelo que propusemos (Multiperiod Hub Location Problem with Serial Demand) foi a de poder trabalhar com frequências para a demanda. Por exemplo, temos que entregar em três vezes a demanda durante 7 dias.

Para a solução desse modelo matemático de otimização, foram utilizadas as técnicas de decomposição de Benders (Benders, 1962), desigualdades válidas foram construídas (combinatória poliédrica) e resolvemos com o software CPLEX 12.8.

Para ilustrar a aplicação desta formulação apresentada em Monemi et al. (2020), considere o exemplo a seguir. Suponha que tenhamos que abastecer durante cinco dias oito campos de refugiados representados pelos nós $1,2, \ldots, 8$. Consideramos o nó zero associado ao depósito principal. Em todos os oito campos, as demandas devem ser entregues em três vezes semanais. Por exemplo, o campo 1 deseja receber $30 \%, 20 \%$ e $50 \%$ da sua demanda semanal, já o campo 2 possui entregas de $10 \%, 60 \%$ e $30 \%$.

A Figura 1 apresenta o resultado do modelo matemático. Na segunda-feira, os nós 1, 2, 3, 4, 5 e 6 são abastecidos e os centros de distribuição foram localizados nos nós 2 e 4 . Na terça-feira os nós 1,4,5,7 e 8 são atendidos e o centro de distribuição foi localizado no nó 7, e assim por diante. Podemos verificar que cada nó foi atendido 3 vezes. Por exemplo, o nó 1 foi abastecido na segunda-feira, na terça-feira e na sexta-feira.

Figura 1: Exemplo de aplicação de Pesquisa Operacional para o abastecimento de um campo de refugiados.

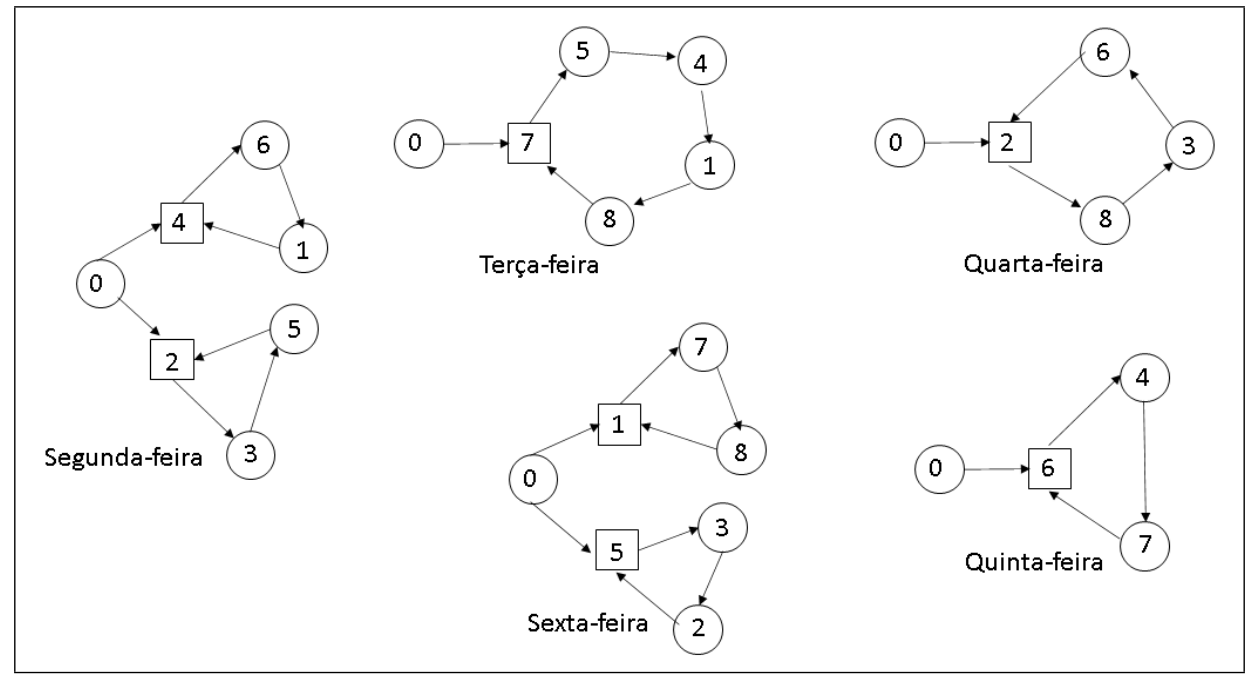

Fonte: Elaborada pelos autores. 


\section{Pandemias}

No manifesto do Prof. Nelson Maulan Filho (Maculan, 2020), publicado em 29 de abril de 2020 no site da IFORS - The International Federation of Operational Research Societies, e na conferência do Prof. Yinyu Ye (Ye, 2020), realizada em 15 de maio de 2020 na Universidade de Stanford, já podemos ter uma boa ideia de onde a Pesquisa Operacional pode atuar de maneira exemplar visando minimizar as perdas humanas em um contexto de pandemia.

Alguns países foram muito atingidos pelo novo coronavírus, outros já nem tanto, alguns estão na fase aguda da pandemia, e outros esperam pela tão temida segunda onda. Para atender as demandas de sobrevivência de diferentes camadas da sociedade humana, que precisam viver no confinamento, há necessidade de criar serviços de distribuição de bens essenciais: comida, medicamentos, bujões de gás, roupas, eletricidade, água etc. Também há de se manter os serviços de saúde, de manutenção das redes (eletricidade, água, gás, telecomunicação etc.) e de segurança (polícia, bombeiros). Sem nos esquecer da educação e cultura, usando as vias de comunicação à distância (televisão, internet etc.). Evitar a aglomeração de pessoas é a melhor maneira de conter a propagação da COVID-19 para que os hospitais possam atender as pessoas com os sintomas mais fortes dessa doença. Tudo isso em tempo real.

Nós que estamos envolvidos nas áreas de pesquisa operacional, matemática aplicada, ciência da computação, estatística, engenharias e áreas afins temos que nos organizar visando propor soluções para as comunidades, os municípios, os estados e a união. Nossas instituições de educação superior, além do trabalho que já vêm fazendo nos mais diversos campos da pesquisa sobre a doença, têm trabalhado também no suprimento de materiais e têm assessorado prefeituras, governos estaduais e o governo federal. No Brasil, as sociedades científicas SOBRAPO, SBMAC, SBC, SBPC, ABC, ANE e outras do ramo podem também, através de suas representações regionais, auxiliar o poder constituído local para propor possíveis soluções para os problemas de transporte e de localização de novos locais de atendimento e de distribuição de bens essenciais.

Um dos setores mais sensíveis atingido pelo confinamento é, sem dúvida, o ensino público, sobretudo na educação básica. Crianças poderão carregar para a vida adulta os prejuízos cognitivos e de sociabilização causados pela interrupção escolar de muitos meses. Há problemas federais, estaduais, municipais e comunitários. Podemos atuar em todos esses níveis.

Além dos modelos de previsão da evolução do número de casos de pessoas infectadas pelo vírus SARS-CoV-2 e do número de mortes provocadas pela COVID-19, as respostas no menor tempo possível para o atendimento são essenciais para salvar vidas.

Podemos nos basear nos trabalhos do Professor Marcos Negreiros da Universidade Estadual do Ceará, Fortaleza, que é pioneiro na aplicação de Pesquisa Operacional para minimizar os danos de epidemias da dengue. Desde 1996, ele se dedica intensamente em construir e aperfeiçoar seu WebDengue (Framework Computacional para Gestão e Apoio à Decisão na Prevenção e Controle de Arboviroses provocadas pelo Aedes Aegypt), ver (Negreiros et al., 2011, 2020).

Marcos Negreiros iniciou trabalhando e propondo formulações matemáticas para problemas de otimização de percurso (roteamento) em redes e suas implementações computacionais. Quase todos esses problemas são NP-difíceis, logo respostas em tempo computacional razoável poderiam ser impossíveis sem o uso de algumas heurísticas e metaheurísticas.

Havia também o problema de como organizar os dados, dentre os quais muitos associados aos aspectos geográficos. Como consequência, ele propôs representações gráficas dinâmicas das soluções e introduziu modelos estatísticos visando à elaboração de cenários para a evolução da endemia.

Seus grandes colaboradores foram o Professor Adilson Xavier, da Universidade Federal do Rio de Janeiro, e o saudoso Professor Airton Xavier, da Universidade Federal do Ceará. Sem contar os estudantes que fizeram suas dissertações de mestrado sob a orientação do Professor Marcos Negreiros.

Em seu trabalho Negreiros et al. (2011) temos uma ideia geral das suas contribuições até 2010. Marcos Negreiros trouxe novas ferramentas de tomada de decisão para combater o mosquito 
Aedes Aegypt e muito mais continua fazendo até hoje.

O framework computacional que ele desenvolveu é formado por um conjunto de seis sistemas computacionais (Webdengue, Gestor, Geographvs, Dynagraph, Móvel Supervisor, e Módulo Móvel Agente) que interagem entre si, com o objetivo de tratar de forma integrada e rápida as informações relacionadas à operação do controle e combate, e ao acompanhamento da evolução da doença em humanos.

A Figura 2 ilustra esse framework, em que Sinan representa o Banco de dados do Ministério da Saúde (SINAN, 2020), LACEN é a sigla para os laboratórios de saúde do sistema nacional (LACEN, 2020), SisPNCD é o Sistema do Programa Nacional de Controle da Dengue desenvolvido pelo Ministério da Saúde, as siglas SVS-MS representam Secretaria da Vigilância da Saúde (SVS) e Ministério da Saúde (MS), respectivamente, e Api R é uma biblioteca de recursos da linguagem estatística $\mathrm{R}$, disponibilizada para ser usada com programas na web.

Maiores detalhes podem ser encontrados em Negreiros et al. (2020). Esse framework poderá ser a base de um novo sistema para minimizar as perdas humanas no período de pandemia provocada pelo novo cornavírus SARS-CoV-2 (responsável pela doença COVID-19).

Figura 2: WebDengue: Framework Computacional para Gestão e Apoio à Decisão na Prevenção e Controle de Arboviroses provocadas pelo Aedes Aegypt.

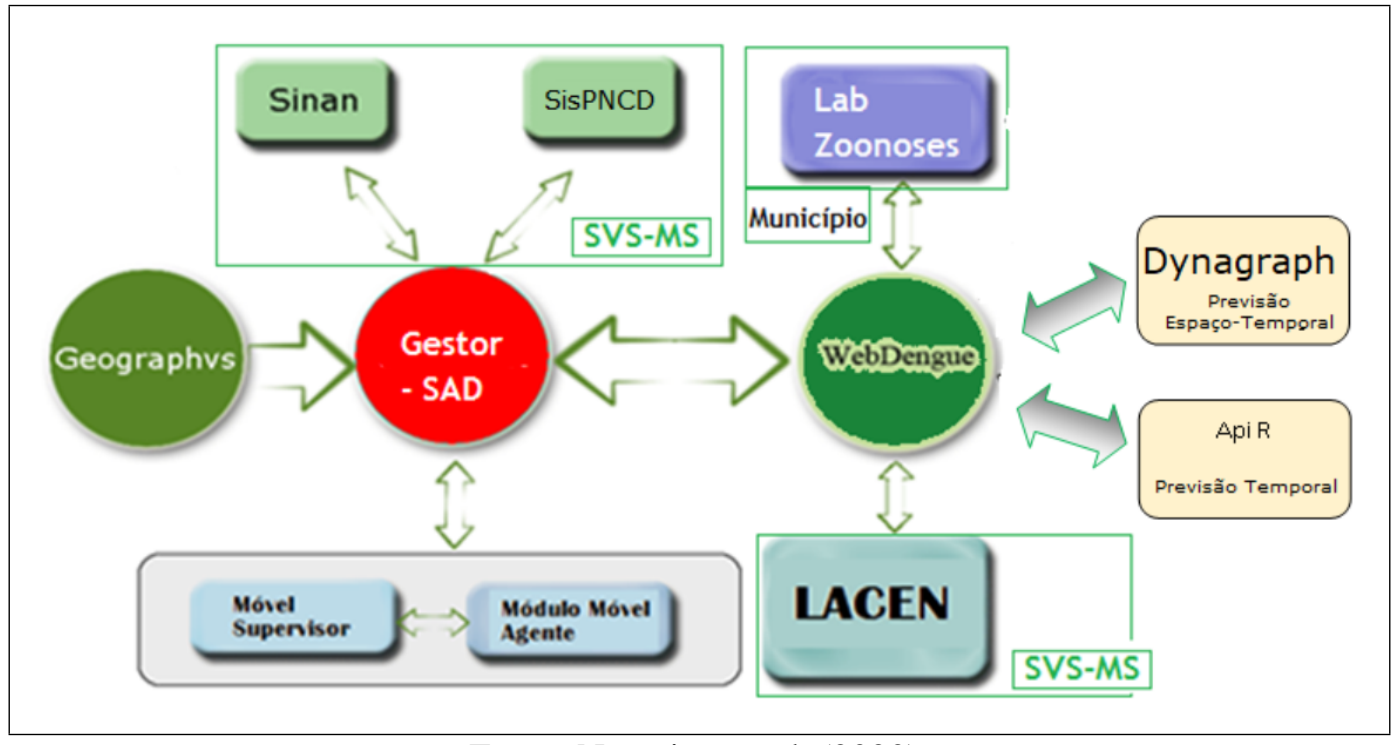

Fonte: Negreiros et al. (2020).

\section{Catástrofes Naturais}

A incidência de eventos naturais, como furacões, terremotos, tsunamis, deslizamentos de terra e enchentes, vem crescendo nos últimos anos, conforme aumentam as alterações das condições climáticas e as ocupações desordenadas do solo promovidas pelo homem. Quando esses eventos acontecem em regiões habitadas, costumam ser chamados de desastres naturais, e podem se tornar ainda mais catastróficos em regiões populosas, com grande risco de dizimação da população.

Em cenários de desastres ou catástrofes naturais, as tomadas de decisão e as respostas logísticas para a mitigação dos efeitos danosos à população, à economia e ao meio-ambiente têm que ser muito rápidas, além de eficientes e eficazes. Esse tratamento logístico especial é chamado na literatura de logística humanitária.

As operações logísticas humanitárias devem ser iniciadas imediatamente após uma catástrofe natural, de modo a prestar rápida assistência às vítimas, no que tange à remoção de mortos, resgate 
de feridos, assistência médica, fornecimento de água potável, alimentos e outros suprimentos, e estruturação de centros de atendimento e acolhimento.

Atrasos nas operações logísticas para resposta a desastres naturais podem levar à perda de muitas vidas, o que torna o gerenciamento dessas operações um grande desafio para os tomadores de decisão. Em consequência, acadêmicos e profissionais da área de pesquisa operacional vêm buscando desenvolver ferramentas eficientes e eficazes para apoiar os processos de tomada de decisão em cenários de catástrofe natural.

Um fator comum e muito importante no contexto de desastres naturais é a destruição da infraestrutura física do ambiente afetado, tornando a eficiência logística extremamente crítica para o sucesso das operações humanitárias.

Desta maneira, os conceitos já bem consolidados na logística tradicional devem ser adaptados às especificidades da logística humanitária. Por exemplo, em relação à infraestrutura, se o transporte terrestre for destruído, transportes alternativos devem ser utilizados ou equipamentos específicos devem ser empregados para desbloquear as estradas para garantir a chegada de recursos e a saída de pessoas; no que diz respeito aos recursos humanos, é fundamental que tenham treinamento adequado à situação em questão; no tocante aos suprimentos, é importante assegurar que sejam entregues de forma contínua ao longo do período afetado.

Contudo, para que as operações humanitárias sejam bem sucedidas, são fundamentais o planejamento, a coordenação, e o controle dos fluxos entre todos os processos e agentes envolvidos nessa complexa cadeia logística.

Quando há falhas, pode haver excesso, falta ou inadequação na distribuição dos suprimentos (quando há falta, pessoas podem morrer; quando há excesso, pode haver deterioração, roubo ou comercialização irregular; no caso da inadequação, as doações podem ficar muito concentradas em um tipo de material, muitas vezes não tão essencial); presença de voluntários despreparados; sobreposição de esforços para atuação em um mesmo problema; desperdício ou falta de recursos financeiros e equívocos no dimensionamento dos centros de acolhimento (os pequenos costumam funcionar melhor, com atendimento mais personalizado, porém também geram aglomeração, que pode gerar violência e contribuir para a propagação de doenças); dentre outros.

O planejamento, a coordenação, e o controle dos fluxos da cadeia logística humanitária estão sujeitos a mudanças constantes ao longo do duração da catástrofe natural, uma vez que o próprio ambiente está sujeito a alterações imprevisíveis.

Essas características são típicas dos sistemas complexos, que costumam possuir as seguintes características: são dinâmicos (evoluem no tempo), altamente acoplados (suas partes interagem entre si), adaptativos (seus agentes aprendem ao longo do tempo, alteram sua percepção e geram ações adaptadas a novos estados que podem surgir no sistema), não lineares (não verificam a proporcionalidade e a linearidade entre as relações de causa e efeito), não markovianos (possuem memória), e auto-organizados (as interações entre as partes do sistema geram comportamentos endógenos), fazendo com que os planos de ação devam ser constantemente revistos.

Levantamentos bibliográficos sobre os principais métodos de pesquisa operacional utilizados no suporte às decisões envolvendo a logística humanitária em casos catástrofes naturais foram realizados por Altay e Green (2006), Galindo e Batta (2013), Marinho et al. (2018) e Díaz et al. (2019). Como conclusão, programação matemática, programação estocástica, teoria da decisão, análise conceitual, simulação e dinâmica de sistemas foram os mais utilizados, com um crescimento recente de métodos baseados também em data mining e machine learning.

Segundo Marinho et al. (2018), a grande maioria dos trabalhos encontrados na revisão bibliográfica considerou dados provenientes de desastres reais para o desenvolvimento de seus modelos e/ou técnicas de pesquisa operacional, contudo sem aplicar estas técnicas em algum desses desastres na prática.

Por exemplo, Pinto e Oliveira (2014) desenvolveu modelos de simulação (baseados em agentes e eventos discretos) e otimização capazes de representar o serviço de atendimento do Corpo de Bombeiros aos efeitos dos fenômenos hidrológicos mais recorrentes na cidade de Belo 
Horizonte. Para tal, utilizaram grande quantidade de dados reais de monitoramento hidrológico e de registros de chamadas de emergência.

Em Brito Jr. (2015), uma metodologia baseada em modelos de otimização estocástica de dois estágios e análise de decisão multicritério foi proposta para a localização de depósitos de suprimentos utilizados no socorro a populações mais sujeitas a serem afetadas por desastres, em uma parceria com a Defesa Civil do Estado de São Paulo.

Já em Marinho et al. (2018), um modelo de localização de centros de auxílio e distribuição de suprimentos foi proposto para auxiliar nas decisões logísticas das fases de preparação e resposta humanitária a desastres ambientais. Os autores criaram uma ferramenta simples de apoio à tomada de decisão - baseada em planilha eletrônica - e usando o modelo proposto, e testaram sua aplicação aos dados reais da catástrofe ocorrida na Região Serrana do Rio de Janeiro em 2011. Um aspecto interessante é que a linguagem de modelagem e os softwares utilizados pelos autores são de uso livre, o que garante o uso da ferramenta em situações de desastre sem a necessidade de gastos adicionais com aquisição de software comercial.

Por fim, a pesquisa operacional e a inteligência computacional também podem ser aplicadas para aferir o impacto das catástrofes naturais no meio-ambiente.

Por exemplo, Cordeiro et al. (2019) avaliaram os impactos provenientes da ruptura da barragem de Fundão em Mariana, em Minas Gerais, em 2015, na qualidade da água do rio Doce. Essa catástrofe ambiental liberou 40 milhões de metros cúbicos de resíduos, varrendo comunidades inteiras, contaminando rios e matando pessoas. Foram analisados os parâmetros metagenômicos e físico-químicos durante o mês do desastre e novamente seis e dez meses após o desastre. Os impactos a curto prazo nas comunidades microbianas incluíram aumento da virulência microbiana e do metabolismo de ferro e nitrogênio, sugerindo alterações nos perfis metabólicos microbianos. Além disso, um ano após o desastre, na estação chuvosa, o rio Doce ainda apresentava turbidez anormal. A Figura 3 ilustra a metodologia utilizada para avaliar esses impactos ambientais.

Figura 3: Metodologia utilizada para avaliar os impactos provenientes da ruptura da barragem de Fundão em Mariana/MG-2015 na qualidade da água do rio Doce.

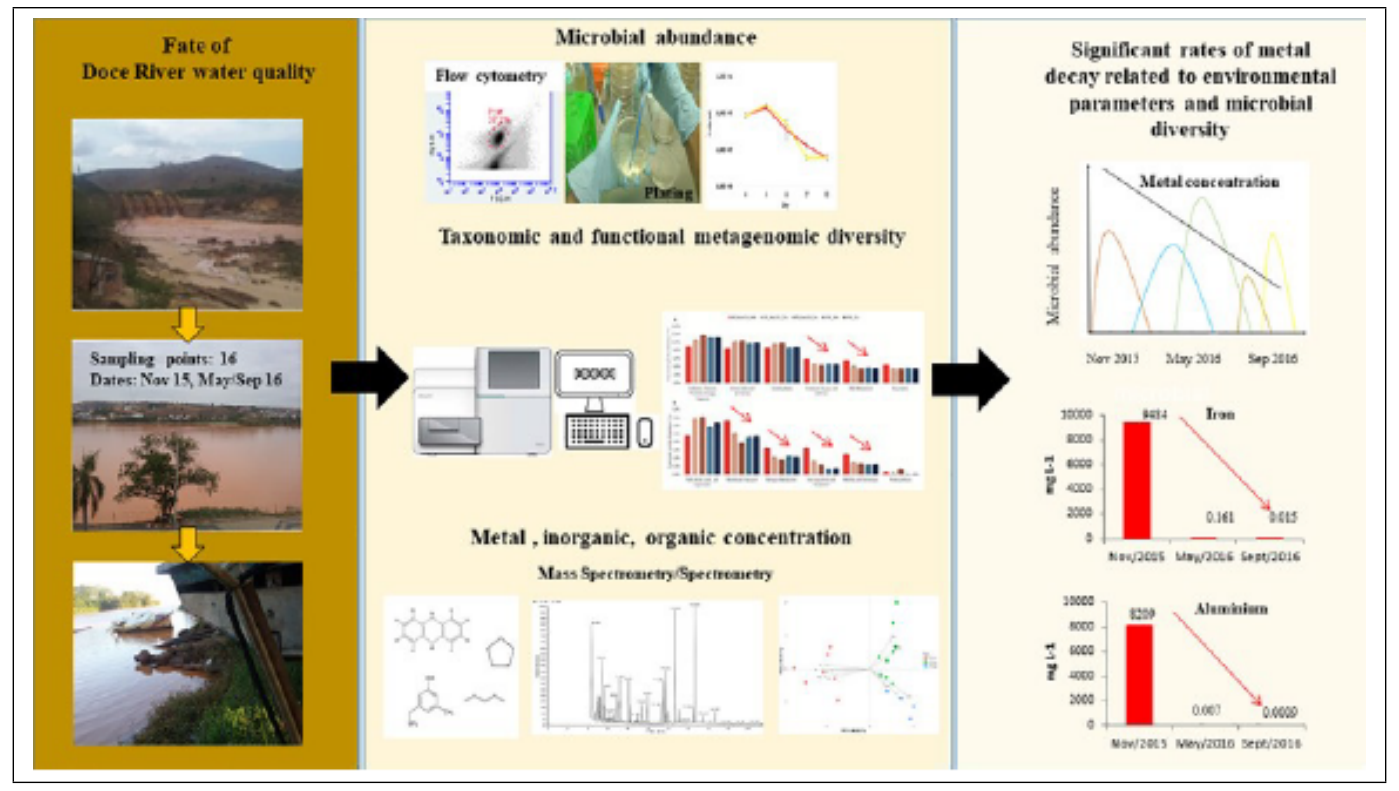

Fonte: Cordeiro et al. (2019).

Ainda sobre o desastre de Mariana, Francini-Filho et al. (2019) mostraram que a lama de rejeitos de minério proveniente da ruptura da barragem de Fundão chegou aos ecossistemas recifais do sul do Banco de Abrolhos, e analisaram os possíveis impactos de curto prazo na biodiversidade marinha destes importantíssimos ecossistemas. A Figura 4 ilustra a metodologia utilizada para avaliar esses impactos ambientais. 
Figura 4: Metodologia utilizada para avaliar os impactos provenientes da ruptura da barragem de Fundão em Mariana/MG-2015 na biodiversidade marinha dos ecossistemas recifais do sul do Banco de Abrolhos.

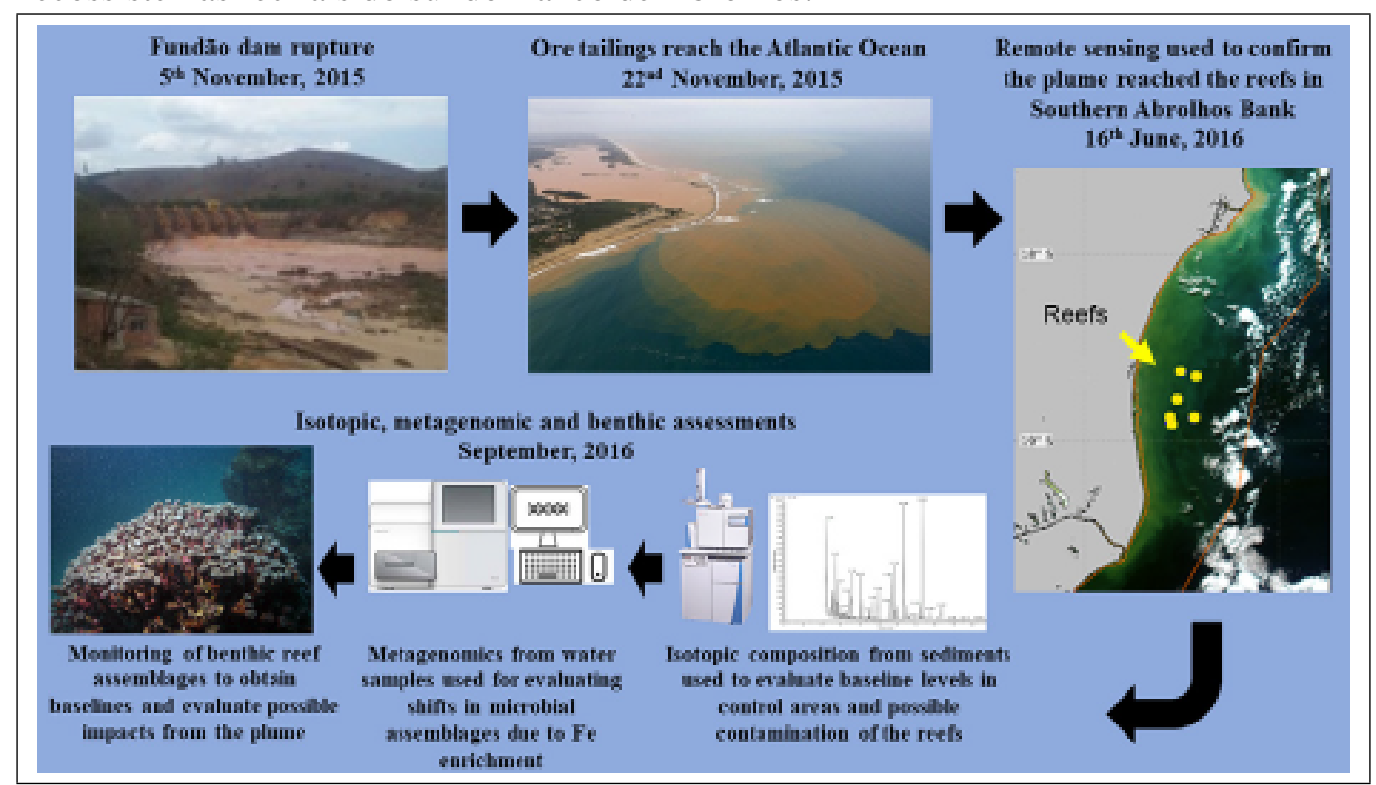

Fonte: Francini-Filho et al. (2019).

Agradecimentos. Os autores agradecem ao Conselho Nacional de Desenvolvimento Científico e Tecnológico - CNPq, bolsas de produtividade em pesquisa \#302435/2019-0 e \#309661/2019-6.

\section{Referências}

Altay, N. e Green, W. G. OR/MS research in disaster operations management. European Journal of Operational Research, v. 175, n. 1, p. 475-493, 2006.

Benders, J. F. Partitioning procedures for solving mixed-variables programming problems. Numerische Mathematik, v. 4, p. 238-252, 1962.

Brito Jr., I. Localização de depósitos de suprimentos de alivio para resposta a desastres através de programação linear estocástica e análise de decisão com múltiplos critérios. $234 \mathrm{f}$. Tese (Doutorado em Engenharia de Produção) - Escola Politécnica, Universidade de São Paulo, São Paulo-SP, 2015.

Camacho-Vallejo, J. F., González-Rodríguez, E., Almaguer, F.-J. e González-Ramírez, R. G. A bi-level optimization model for aid distribution after the occurrence of a disaster. Journal of Cleaner Production, v. 105, p. 134-145, 2015.

Cordeiro, M. C., Garcia, G. D., Rocha, A. M., Tschoeke, D. A., Campeão, M. E., Appolinario, L. R., et al. Insights on the freshwater microbiomes metabolic changes associated with the world's largest mining disaster. Science of The Total Environment, v. 654, p. 1209-1217, 2019.

Díaz, H. L., Imitola, K. A. e Amado, R. J. A. OR/MS research perspectives in disaster operations management: a literature review. Revista Facultad de Ingeniería Universidad de Antioquia, v. 91, p. 43-59, 2019.

Fereiduni, M., Hamzehee, M. e Shahanaghi, K. A robust optimization model for logistics planning in the earthquake response phase. Decision Science Letters, v. 5, p. 519-534, 2016. 
Francini-Filho, R. B., Cordeiro, M. C., Omachi, C. Y., Rocha, A. M., Bahiense, L., Garcia, et al. Remote sensing, isotopic composition and metagenomics analyses revealed Doce River ore plume reached the southern Abrolhos Bank Reefs. Science of The Total Environment, v. 697, p. 134038, 2019.

Galindo, G. e Batta, R. Review of recent developments in OR/MS research in disaster operations management. European Journal of Operational Research, v. 230, n. 2, p. 201-211, 2013.

LANCEN - Laboratório Central de Saúde Pública. Laboratórios de Saúde do Sistema Nacional. 2020. Disponível em: https://www.saude.gov.br/acoes-e-programas/. Acesso em: 25/06/2020.

Maculan, N. Manifesto. 2020. Disponível em: https://www.ifors.org/manifesto-nelson-maculan/. Acesso em: 26/06/2020.

Maculan-Guido. Modelo de Programação Linear para Alocar Recursos em Vias e Hospitais em Caso de Desastre. 82 f. Dissertação (Mestrado em Engenharia de Produção) - Departamento de Engenharia Industrial, Pontifícia Universidade Católica do Rio de Janeiro, Rio de Janeiro-RJ, 2017.

Marinho, F., Munari, P., Moreno, A. e Alem, D. Uma ferramenta baseada em otimização para apoiar decisões de preparação e resposta em logística humanitária. Pesquisa Operacional para o Desenvolvimento, v. 10, n. 2, p. 70-92, 2018.

Monemi, R. N., Gelareh, S., Nagih, A., Maculan, N. e Danache, K. Multi-period hub location problem with serial demands: A case study of humanitarian aids distribution in lebanon. Relatório Técnico em Engenharia de Sistemas e Computação, ES-762/20, COPPE, Universidade Federal do Rio de Janeiro, p. 1-30, https://cos.ufrj.br/index.php/pt-BR/publicacoes-pesquisa/details/15/2949, 2020.

Nagurney, A. Perishable food supply chain networks with labor in the covid-19 pandemic. In: Kotsireas, I., Nagurney, A., e Pardalos, P. (eds.), Dynamics of Disasters - Impact, Risk, Resilience, and Solutions. Springer International Publishing Switzerland, 2020.

Negreiros, M. J., Maculan, N. e Xavier, A. E. Projeto Webdengue: evolução e recursos de implantação. Relatório Técnico em Engenharia de Sistemas e Computação, ES-763/20, https://cos.ufrj.br/index.php/pt-BR/publicacoes-pesquisa/details/15/2951, COPPE, Universidade Federal do Rio de Janeiro, 2020.

Negreiros, M., Xavier, A. E., Xavier, A. F. S., Maculan, N., Michelon, P., Lima, J. W. O. e Andrade, L. O. M. Optimization models, statistical and DSS tools for prevention and combat of dengue disease. In: S.Jao, C., (ed.), Efficient Decision Support Systems: Practice and Challenges in Biomedical Related Domain, p. 115-160. Croatia: IntechOpen, 2011.

Pinto e Oliveira, B. R. Simulação dos serviços de atendimento aos efeitos gerados pelos fenômenos hidrológicos mais recorrentes em Belo Horizonte. $83 \mathrm{f}$. Dissertação (Mestrado em Engenharia de Produção) - Departamento de Engenharia de Produção, Universidade Federal de Minas Gerais, Belo Horizonte-MG, 2014.

SINAN - Sistema de Informação de Agravos de Notificação. Banco de dados do ministério da saúde. 2020. Disponível em: http://portalsinan.saude.gov.br/. Acesso em: 25/06/2020.

Ye, Y. Optimization and operations research in mitigation of a pandemic. 2020. Disponível em: https://web.stanford.edu/ yyye/ORapplicationsinPandemic.pdf. Acesso em: 25/06/2020. 